\title{
晶粒互锁结构与短切碳纤维增韧 $\mathrm{ZrB}_{2}-\mathrm{SiC}$ 复合材料的制备与力学性能
}

\begin{abstract}
张兆甫 ${ }^{1}$, 沙建军 ${ }^{1,2}$, 祖宇飞 ${ }^{1}$, 代吉祥 ${ }^{1}$
(大连理工大学 1. 辽宁省空天飞行器前沿技术重点实验室; 2. 航空航天学院, 工业装备结构分析国家重点实验室, 大连 116024)

摘 要: $\mathrm{ZrB}_{2}-\mathrm{SiC}$ 基复合材料具有比单体 $\mathrm{ZrB}_{2}$ 更优异的抗氧化性能及力学性能, 但其相对较低的韧性限制了其实际 工程应用, 采用微结构设计或引入增韧相是改善陶瓷材料韧性的两个有效途径。本研究采用反应热压烧结工艺, 分 别制备了具有独特片状 $\mathrm{ZrB}_{2}$ 晶粒互锁结构的 $\mathrm{ZrB}_{2}-\mathrm{SiC}$ 复合材料和以短切碳纤维 $\left(\mathrm{C}_{\mathrm{sf}}\right)$ 为增韧相的 $\mathrm{C}_{\mathrm{sf}} / \mathrm{ZrB}_{2}-\mathrm{SiC}$ 复合 材料。对比研究发现, 晶粒互锁结构展现出优异的自强韧化效果, 使 $\mathrm{ZrB}_{2}-\mathrm{SiC}$ 复合材料具有较高的弯曲强度及断 裂韧性, 但材料表现出典型的脆性断裂特征; $\mathrm{C}_{\mathrm{sf}} / \mathrm{ZrB}_{2}-\mathrm{SiC}$ 复合材料弯曲强度下降, 但 $\mathrm{C}_{\mathrm{sf}}$ 具有显著的增㓞作用, 不 仅使材料具有较高的断裂韧性，而且临界裂纹尺寸及断裂功都得到显著提高，从而表现出非灾难性破坏模式。
\end{abstract}

关 键 词: 超高温陶瓷; 晶粒互锁结构; 短切碳纤维; $\mathrm{ZrB}_{2}-\mathrm{SiC}$; 断裂性能

中图分类号: TB332 文献标识码: A

\section{$\mathrm{ZrB}_{2}$-SiC Composites Toughened by Interlocking Microstructure and Chopped Carbon Fiber}

\begin{abstract}
ZHANG Zhao-Fu ${ }^{1}$, SHA Jian-Jun ${ }^{1,2}$, ZU Yu-Fei $^{1}$, DAI Ji-Xiang ${ }^{1}$
(1. Key Lab of Advanced Technology for Aerospace Vehicles, Dalian University of Technology, Dalian 116024, China; 2. State Key Lab. of Structural Analyses for Industrial Equipment, School of Aeronautics and Astronautics, Dalian University of Technology, Dalian 116024, China)
\end{abstract}

\begin{abstract}
ZrB}_{2}-\mathrm{SiC}$ ceramics present better oxidation resistance and mechanical properties than monolithic $\mathrm{ZrB}_{2}$ ceramics. However, the small damage tolerance and poor crack growth resistance, which result in the low fracture toughness, limit the engineering application of $\mathrm{ZrB}_{2}-\mathrm{SiC}$ ceramics. Focusing on this issue, microstructure design and introduction of toughening phase are two effective approaches to improve the fracture toughness of $\mathrm{ZrB}_{2}-\mathrm{SiC}$ ceramics. In this work, $\mathrm{ZrB}_{2}-\mathrm{SiC}$ and $\mathrm{C}_{\mathrm{f}} / \mathrm{ZrB}_{2}-\mathrm{SiC}$ composites were toughened respectively by interlocking microstructure and chopped carbon fibers via reactive hot pressing. For the $\mathrm{ZrB}_{2}-\mathrm{SiC}$ composites, the interlocking microstructure formed by in-situ $\mathrm{ZrB}_{2}$ platelets presented excellent self-enhancing effect. The $\mathrm{ZrB}_{2}$-SiC composites had high bending strength and fracture toughness. However, the composite exhibited typical brittle fracture characteristics. Compared with $\mathrm{ZrB}_{2}-\mathrm{SiC}$ composite, the flexural strength of $\mathrm{C}_{\mathrm{f}} / \mathrm{ZrB}_{2}-\mathrm{SiC}$ composite decreased, but the fracture toughness was comparable with the $\mathrm{ZrB}_{2}-\mathrm{SiC}$ composite. Furthermore, the critical crack size and the work of fracture of $\mathrm{C}_{\mathrm{f}} / \mathrm{ZrB}_{2}$-SiC composites significantly improved, and the composite presented the non-catastrophic failure mode.
\end{abstract}

收稿日期: 2018-11-28; 收到修改稿日期：2019-01-22

基金项目: 国家自然科学基金(51805069); 中国博士后科学基金(2016M600201，2018T110214,2016M601304); 辽宁省自然 科学基金(20170540154); 航空科学基金(2016ZF63007)

National Natural Science Foundation of China (51805069); China Postdoctoral Science Foundation (2016M600201, 2018T110214, 2016M601304); Natural Science Foundation of Liaoning Province, China (20170540154); Aviation Science Foundation of China (2016ZF63007)

作者简介: 张兆甫(1990-), 男, 博士研究生. E-mail: zhangzhaofu@mail.dlut.edu.cn

通讯作者: 沙建军, 教授. E-mail: jjsha@dlut.edu.cn 
Key words: ultra-high temperature ceramic; interlocking microstructure; chopped carbon fiber; $\mathrm{ZrB}_{2}-\mathrm{SiC}$; fracture property

近年来空间飞行器技术和现代工业的快速发展, 对具有耐高温、抗氧化及抗粒子侵蚀等极端环境材 料的需求不断提高, 使超高温陶瓷(Ultra-high Temperature Ceramics, UHTC)材料越来越受到关注 ${ }^{[1]}$ 。其中, 二硼化锆 $\left(\mathrm{ZrB}_{2}\right)$ 因较低的密度以及优异的耐热环境 性能, 成为新型航天器热端结构、工业耐火祄里和切 削工具等最具潜力的候选材料 ${ }^{[2-3]}$ 。特别是 $\mathrm{ZrB}_{2}-\mathrm{SiC}$ 超高温陶瓷体系, 不仅具有比单体 $\mathrm{ZrB}_{2}$ 陶瓷更优异 的烧结性能, 而且表现出更优异的抗氧化和高温力 学性能, 成为超高温材料研究领域受到广泛关注的 体系 ${ }^{[4]}$ 。然而, 由于陶瓷材料的固有脆性, 使得 $\mathrm{ZrB}_{2}-\mathrm{SiC}$ 材料的断裂韧性一般在 3.5 5.0 MPa $\mathrm{m}^{1 / 2}$, 容 易在缺陷处诱发裂纹失稳而导致灾难性破坏, 这也 是限制 $\mathrm{ZrB}_{2}-\mathrm{SiC}$ 陶瓷工程化应用的主要问题之一。

降低陶瓷材料对缺陷或裂纹的敏感性, 改善材 料抵抗断裂的性能, 主要有两种途径: 一种是通过 设计获得自增㓞微结构, 另一种是引入增韧相进行 复合化。针对过渡金属硼化物 $\left(\mathrm{MB}_{2}\right)$ 的晶体特征, Zhang 等 ${ }^{[5]}$ 提出了通过促进晶粒各向异性生长来实 现 $\mathrm{MB}_{2}$ 超高温陶瓷微结构设计的概念。 $\mathrm{MB}_{2}$ 晶粒各 向异性生长会形成棒状或片状的自增韧微结构, 在 外载荷的作用下可以引发裂纹偏折及桥接机制, 从 而显著改善材料的强㓞性。 $\mathrm{Wu}$ 等 ${ }^{[6]}$ 制备了 $\mathrm{ZrB}_{2}-\mathrm{MoSi}_{2}$ 复合材料, 发现原位生成的棒状 $\mathrm{ZrB}_{2}$ 晶粒形成了部 分织构化结构, 可以显著改善材料的断裂韧性。另 一方面, 在陶瓷材料中引入碳纳米管 ${ }^{[7]} 、 \mathrm{SiC}$ 晶须 ${ }^{[8]}$ 或短切纤维 ${ }^{[9]}$ 等高长径比的增韧相, 则可以有效提 高材料的损伤容限, 降低材料对缺陷的敏感性。其 中, 短切碳纤维 $\left(\mathrm{C}_{\mathrm{sf}}\right)$ 作为微米尺度的增韧相, 具有 优异的高温稳定性, 易于启动纤维脱粘、拔出以及 桥接等能量耗散机制, 可有效抑制基体缺陷的体积 效应，在改善陶瓷材料灾难性破坏方面具有一定的 优势。Gui 等 ${ }^{[10]}$ 采用热压烧结工艺制备了 $\mathrm{ZrB}_{2}-\mathrm{SiC}_{-} \mathrm{C}_{\mathrm{f}}$ 复合材料, 材料表现出优异的力学性能和抗热冲击 性能，显著优于传统的 $\mathrm{ZrB}_{2}$ 基复合材料。而 $\mathrm{He}$ 等 ${ }^{[11]}$ 采用 $\mathrm{C}_{\mathrm{sf}}$ 增㓞制备的 $\mathrm{C}_{\mathrm{sf}} / \mathrm{Al}_{2} \mathrm{O}_{3}-\mathrm{BN}$ 复合材料在破坏 过程中也展现出典型的㓞性断裂模式。

因此，在 $\mathrm{ZrB}_{2}-\mathrm{SiC}$ 陶瓷体系中，探究合适的烧 结工艺以实现自增㓞微结构或引入 $\mathrm{C}_{\mathrm{sf}}$ 实现复合化, 都将是提升该类材料抵抗破坏能力的有效途径。在 此基础上研究这两种增韧方式对复合材料力学性能 及断裂行为的影响, 对于理解 $\mathrm{ZrB}_{2}-\mathrm{SiC}$ 陶瓷复合材
料的增韧机制具有重要意义。为此, 本研究采用原 位反应热压烧结工艺制备了具有特殊自强韧微结构 的 $\mathrm{ZrB}_{2}-\mathrm{SiC}$ 复合材料, 同时引入 $\mathrm{C}_{\mathrm{sf}}$ 作为增韧相, 制 备了 $\mathrm{C}_{\mathrm{s}} / \mathrm{ZrB}_{2}-\mathrm{SiC}$ 复合材料, 对比研究了自强韧微 结构与 $\mathrm{C}_{\mathrm{sf}}$ 对 $\mathrm{ZrB}_{2}-\mathrm{SiC}$ 陶瓷断裂性能的影响, 探讨 了相关的增韧机理。

\section{1 实验方法}

\section{1 材料制备}

原位反应热压烧结所采用的原料为: $\mathrm{ZrSi}_{2}$ (平均 粒径为 $5 \mu \mathrm{m}$, 纯度 $>99 \%$ )、 $\mathrm{B}_{4} \mathrm{C}$ (粒径为 $2 \sim 3 \mu \mathrm{m}$, 纯 度>99\%)、炭黑(粒径为 $0.1 \sim 1.0 \mu \mathrm{m}$ )、短切碳纤维(日 本东丽公司 $\mathrm{T} 700$, 平均直径 $7 \mu \mathrm{m}$, 平均长度约为 $1 \mathrm{~mm}$ )。

$\mathrm{ZrB}_{2}-\mathrm{SiC}$ 的合成是基于如下反应(1)进行:

$$
2 \mathrm{ZrSi}_{2}+\mathrm{B}_{4} \mathrm{C}+3 \mathrm{C}=2 \mathrm{ZrB}_{2}+4 \mathrm{SiC}
$$

首先将 $\mathrm{ZrSi}_{2} 、 \mathrm{~B}_{4} \mathrm{C}$ 及炭黑以摩尔比 $2.72: 1.00: 3.00$ 配料。对比于反应(1), 本研究在混合粉体中引入过 量的 $\mathrm{ZrSi}_{2}$ 作为烧结助剂。根据反应(1), 当反应进行 完全，则制备得到的复合材料中将存在 $\mathrm{ZrB}_{2} 、 \mathrm{SiC}$ 及 $\mathrm{ZrSi}_{2}$ 三相，其体积含量分别为 $34 \% 、 46 \%$ 及 $20 \%$ 。 将此粉体与无水乙醇按体积比 $1: 1$ 混合, 装入硬质 合金球磨罐中, 采用高能行星式球磨机进行球磨混 合, 研磨介质为 $\mathrm{WC}$ 磨球, 转速为 $200 \mathrm{r} / \mathrm{min}$, 球磨 时间为 $12 \mathrm{~h}$ 。球磨后粉体经旋转蒸发后干燥备用。

按照 $20 \%$ 体积分数称取定量的 $\mathrm{C}_{\mathrm{sf}}$, 采用湿法工 艺将 $\mathrm{C}_{\mathrm{sf}}$ 与球磨后的原料粉体混合, 工艺过程为: 先 将 $\mathrm{C}_{\mathrm{sf}}$ 加入酚醛树脂溶液中, 超声震荡打开纤维团 聚; 接着向溶液中加入聚乙烯亚胺溶液, 使酚醛树 脂发生交联反应而形成稳定的胶体溶液; 之后在玻 璃棒摚拌下逐渐加入按比例称量的球磨后原料粉体, 之后超声震荡 $30 \mathrm{~min}$ 使 $\mathrm{C}_{\mathrm{sf}}$ 与原料粉体均匀分散于 胶体溶液中; 最后将胶体溶液注入一端放置石亳板 的石英管中, 待干燥后获得均匀分散的复合材料生 坏体。

将复合材料生坏体装入石墨模具并在热压炉中 完成反应烧结。烧结在真空环境 $(<10 \mathrm{~Pa})$ 下进行, 最 终烧结温度为 $1550{ }^{\circ} \mathrm{C}$, 升温速度 $10{ }^{\circ} \mathrm{C} / \mathrm{min}$ 。其中 在 $1300{ }^{\circ} \mathrm{C}$ 保温 $15 \mathrm{~min}$ 以促进反应进行, 在 1200 、 $1300 、 1400$ 及 $1550{ }^{\circ} \mathrm{C}$ 分别施加 $20 、 25 、 30$ 及 $40 \mathrm{MPa}$ 单轴压力, $1550{ }^{\circ} \mathrm{C}$ 保温加载 $30 \mathrm{~min}$ 完成烧结。文中 
为表述方便, 将所制备的 $\mathrm{ZrB}_{2}-\mathrm{SiC}$ 和 $\mathrm{C}_{\mathrm{sf}}$ 增㓞 $\mathrm{ZrB}_{2}-\mathrm{SiC}$ 陶瓷基复合材料分别简写为: $\mathrm{ZS}$ 及 $\mathrm{C}_{\mathrm{s} f} / \mathrm{ZS}$ 。

\section{2 表征方法}

反应热压后的样品经过表面抛光处理后进行测 试。样品的密度通过阿基米德排水法测定, 理论密 度通过混合法则获得, 并由此计算获得样品的相对 密度。采用 $X$ 射线衍射 $X R D$, 日本岛津 XRD-6000) 对混合粉体及样品进行物相分析; 采用场发射扫描 电镜(FE-SEM, Nova nano SEM450)对样品表面及断 面进行形貌观察。采用维氏硬度计在样品抛光表面 测定复合材料的维氏硬度, 施加载荷为 $5 \mathrm{~kg}$, 保载 时间为 $10 \mathrm{~s}$ 。采用三点弯曲法测定样品的弯曲强度, 试样的尺寸为 $2 \mathrm{~mm} \times 2 \mathrm{~mm} \times 25 \mathrm{~mm}$, 跨距 $18 \mathrm{~mm}$, 加载速率 $0.5 \mathrm{~mm} / \mathrm{min}$; 采用单边缺口梁法(SENB)测 定样品的断裂韧性, 试样的尺寸为 $2 \mathrm{~mm} \times 4 \mathrm{~mm} \times$ $25 \mathrm{~mm}$, 跨距 $18 \mathrm{~mm}$, 加载速率 $0.05 \mathrm{~mm} / \mathrm{min}$; 样品 的断裂功采用公式(2)计算 ${ }^{[12]}$.

$$
\gamma_{\mathrm{wof}}=\frac{W}{2 A}
$$

式中: $W$ 为材料断裂过程中消耗的能量, 可以通过 SENB 测试中计算载荷-位移曲线与横轴间的积分面 积求得; $A$ 为单边缺口梁的有效承载面积。

\section{2 结果与讨论}

\section{1 复合材料的致密化行为}

图 1 为 $\mathrm{ZS}$ 及 $\mathrm{C}_{\mathrm{s}} \mathrm{I} / \mathrm{ZS}$ 复合材料致密化曲线。在 $1200{ }^{\circ} \mathrm{C}$ 以下, 由于仅施加了约 $2 \mathrm{MPa}$ 的预载荷, 复 合材料致密化速率较慢(A 区域)。之后继续施加载 荷, 两样品相对密度迅速提高, 并表现出几乎相同 的致密化速率(B 区域)。进入 $1300{ }^{\circ} \mathrm{C}$ 保温阶段, 载 荷增加至 $25 \mathrm{MPa}$, 两样品致密化速率进一步提高, 并随保温过程的进行而逐渐降低, 但 $\mathrm{C}_{\mathrm{sf}} \mathrm{ZS}$ 致密化 速率低于 $\mathrm{ZS}$, 且下降更快(C 区域)。 $1400{ }^{\circ} \mathrm{C}$ 时, 载 荷升高至 $30 \mathrm{MPa}$, 样品致密化速率再次加快, 但随 温度升高, $\mathrm{ZS}$ 致密化速率继续增大, $\mathrm{C}_{\mathrm{s} f} / \mathrm{ZS}$ 则出现略 微下降(D 区域)。在达到最终烧结温度 $\left(1550{ }^{\circ} \mathrm{C}\right)$ 及 载荷 (40 MPa) 时, 样品 $\mathrm{ZS}$ 通过之前的快速致密化过 程达到较高的相对密度, 从而在保温保载 $5 \mathrm{~min}$ 左 右转入缓慢致密化阶段, 致密化速率逐渐下降, 并 完成烧结过程( $\mathrm{E}$ 区域)。而样品 $\mathrm{C}_{\mathrm{sf}} \mathrm{ZS}$ 在压力达到 $40 \mathrm{MPa}$ 后再次进入快速致密化过程, 致密化速率迅 速增大, 直至最后 $10 \mathrm{~min}$ 左右转入缓慢致密化阶段 完成烧结。

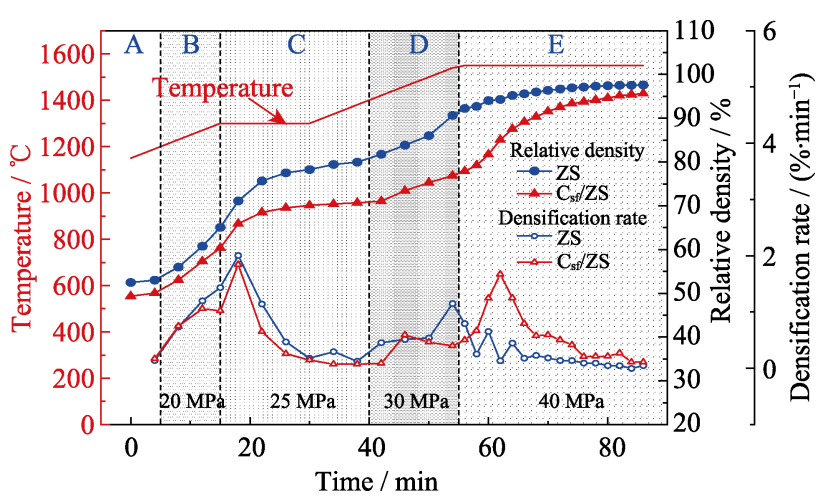

图 $1 \mathrm{ZrB}_{2}-\mathrm{SiC}(\mathrm{ZS})$ 及 $\mathrm{C}_{\mathrm{s} f} / \mathrm{ZrB}_{2}-\mathrm{SiC}\left(\mathrm{C}_{\mathrm{sf}} / \mathrm{ZS}\right)$ 复合材料致密化曲线 Fig. 1 The densification curves of $\mathrm{ZrB}_{2}-\mathrm{SiC}(\mathrm{ZS})$ and $\mathrm{C}_{\mathrm{sf}} / \mathrm{ZrB}_{2}-\mathrm{SiC}\left(\mathrm{C}_{\mathrm{sf}} / \mathrm{ZS}\right)$ composites

由此可见, $\mathrm{C}_{\mathrm{sf}}$ 引入使复合材料的致密化行为出 现了变化。在达到最终烧结压力及温度条件之前, $\mathrm{C}_{\mathrm{sf}} / \mathrm{ZS}$ 的致密化速率低于 $\mathrm{ZS}$ 且下降更快, 直至进入 最终保温保载后 $\mathrm{C}_{\mathrm{sf}} / \mathrm{ZS}$ 才完成快速致密化过程。这 主要是由于 $\mathrm{C}_{\mathrm{sf}}$ 引入后使复合材料坏体成为具有良 好弹性的柱体, 纤维的架桥效应使坏体中粉体接触 面积缩小, 必须通过外部压力克服纤维架桥效应使 粉体产生良好接触, 才能在加热作用下推动复合材 料的快速致密化 ${ }^{[13]}$ 。

$\mathrm{ZS}$ 及 $\mathrm{C}_{\mathrm{sf}} \mathrm{zS}$ 致密化过程的区别也造成了其相 对密度及开孔率的差异, 表 1 列出了 $\mathrm{ZS}$ 及 $\mathrm{C}_{\mathrm{sf}} \mathrm{ZSS}$ 样品的密度、相对密度及孔隙率。可见 $\mathrm{C}_{\mathrm{sf}}$ 的引入 使复合材料的相对密度降低, 这与材料致密化曲线 中观察到的结果相符, 即纤维的架桥效应使材料的 快速致密化阶段延后出现且持续时间缩短, 造成 $\mathrm{C}_{\mathrm{sf}} / \mathrm{ZS}$ 相对密度略微下降。此外, $\mathrm{C}_{\mathrm{sf}} / \mathrm{ZS}$ 样品中开孔 率显著高于闭孔率, 这也是高长径比 $\mathrm{C}_{\mathrm{sf}}$ 在基体中 交错形成联通的通道所造成的。

\section{2 复合材料物相}

图 2 为球磨后混合粉体与反应热压烧结制备样 品的 XRD 图谱。在混合粉体中, $\mathrm{ZrSi}_{2}$ 的峰为主要衍 射峰, $\mathrm{B}_{4} \mathrm{C}$ 的衍射峰较弱, 同时也发现了微弱的 WC 及 $\mathrm{ZrO}_{2}$ 峰。 $\mathrm{ZrO}_{2}$ 的存在主要是球磨前后 $\mathrm{ZrSi}_{2}$ 颗粒 表面的氧化所致, 而 WC 则是球磨过程中由 WC 磨 球引入。根据球磨前后粉体质量变化, 混合粉体中 $\mathrm{WC}$ 含量约为 $5 \mathrm{wt} \%$ 。反应热压烧结后, 两样品中的

表 $1 \mathrm{ZS}$ 及 $\mathrm{C}_{\mathrm{sf}} / \mathrm{ZS}$ 样品密度、相对密度及孔隙率 Table 1 Density, porosity and relative density of ZS and $\mathrm{C}_{\mathrm{sf}} / \mathrm{ZS}$ composites

\begin{tabular}{cccccc}
\hline Sample & $\begin{array}{c}\text { Theoretical } \\
\text { density/ } \\
\left(\mathrm{g} \cdot \mathrm{cm}^{-3}\right)\end{array}$ & $\begin{array}{c}\text { Bulk } \\
\text { density/ } \\
\left(\mathrm{g} \cdot \mathrm{cm}^{-3}\right)\end{array}$ & $\begin{array}{c}\text { Open } \\
\text { porosity/ } / \\
\%\end{array}$ & $\begin{array}{c}\text { Closed } \\
\text { porosity/ } / \\
\%\end{array}$ & $\begin{array}{c}\text { Relative } \\
\text { density/ } \\
\%\end{array}$ \\
\hline $\mathrm{ZS}$ & 4.52 & 4.41 & 1.72 & 0.68 & 97.6 \\
$\mathrm{C}_{\mathrm{sf}} / \mathrm{ZS}$ & 3.97 & 3.79 & 3.27 & 1.13 & 95.6 \\
\hline
\end{tabular}




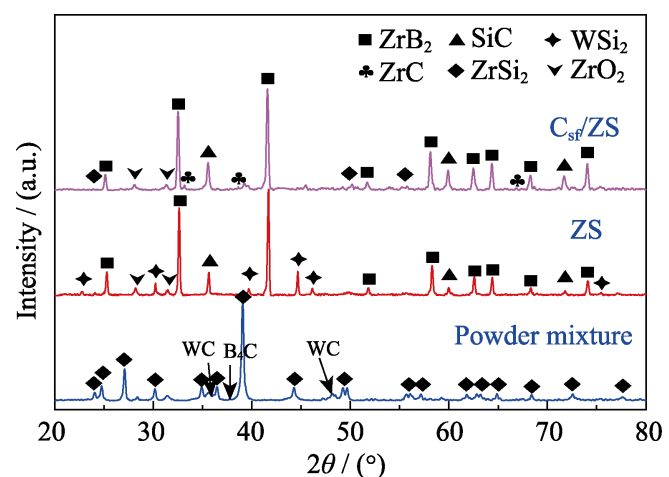

图 2 球磨后混合粉体及反应热压烧结样品的 XRD 图谱

Fig. 2 XRD patterns of ball-milled powder mixture and reactive hot pressed composites

主要结晶相都为 $\mathrm{ZrB}_{2}$ 及 $\mathrm{SiC}$, 同时伴随着微弱的 $\mathrm{ZrSi}_{2}$ 衍射峰, 无其它原料粉体衍射峰存在, 表明烧 结过程是按照设计反应(1)完全进行的。

另外, 在样品 $\mathrm{ZS}$ 中检测出了 $\mathrm{WSi}_{2}$ 相, 而样品 $\mathrm{C}_{\mathrm{s} f} / \mathrm{ZS}$ 中 $\mathrm{WSi}_{2}$ 相衍射峰强度显著降低, 并检测出 $\mathrm{ZrC}$ 相, 这说明两种材料在烧结过程中发生了除反 应(1)以外的其它反应。本研究中引入了过量的 $\mathrm{ZrSi}_{2}$ 作为烧结助剂, 其可能参与如下两个反应 ${ }^{[14-15]}$ :

$$
\begin{gathered}
\mathrm{ZrSi}_{2}+\mathrm{WC}=\mathrm{ZrC}+\mathrm{WSi}_{2} \\
\mathrm{ZrSi}_{2}+3 \mathrm{C}=\mathrm{ZrC}+2 \mathrm{SiC}
\end{gathered}
$$

从反应(3)可以看出, $\mathrm{WSi}_{2}$ 相主要是由于球磨中 引入的微量 $\mathrm{WC}$ 与 $\mathrm{ZrSi}_{2}$ 发生反应所致。此外, 由于 在引入 $\mathrm{C}_{\mathrm{sf}}$ 过程中使用了有机胶体作为分散介质, 将使材料在烧结过程中生成裂解炭, 其具有相对较 高的反应活性，从而促进反应(4)发生，在材料中生 成 $\mathrm{ZrC}$ 。而反应(4)吉布斯自由能(1000 1600 ${ }^{\circ} \mathrm{C}$ : $-159.3 \sim-152.5 \mathrm{~kJ} / \mathrm{mol}$ ) 比反应(3)吉布斯自由能 $\left(1000 \sim 1600{ }^{\circ} \mathrm{C}:-84.9 \sim 84.3 \mathrm{~kJ} / \mathrm{mol}\right)$ 更低, 更容易正 向进行, 最终造成 $\mathrm{C}_{\mathrm{s}} / \mathrm{ZS}$ 中 $\mathrm{ZrC}$ 衍射峰增强而 $\mathrm{WSi}_{2}$ 衍射峰减弱。反应(3)与(4)的发生在一定程度上减少 了材料中 $\mathrm{ZrSi}_{2}$ 烧结助剂的残余, 并且生
成的含 $\mathrm{W}$ 化合物及 $\mathrm{ZrC}$ 有利于 $\mathrm{ZrB}_{2}-\mathrm{SiC}$ 陶瓷抗氧 化及烧蚀性能的提升 ${ }^{[16-17]}$ 。

\section{3 复合材料微观结构}

图 3 为 ZS 样品微观形貌及 EDS 分析结果。在 抛光面背散射电子显微照片(图 3(a)) 中观察到三种 相成分, 分别为灰色的 $\mathrm{ZrB}_{2}$ 相、黑色的 $\mathrm{SiC}$ 相以及 一些分布于 $\mathrm{ZrB}_{2}$ 及 $\mathrm{SiC}$ 之间的白色相。根据图 3(c) EDS 分析结果可知, 白色相主要包含 $\mathrm{Si}$ 及 $\mathrm{W}$ 元素, 原子比接近 $2: 1$, 结合 XRD 分析结果, 其主要为 $\mathrm{WSi}_{2}$ 相。

图 3(b)为 ZS 样品断面微观形貌, 可以看到反应 热压烧结生成的 $\mathrm{ZrB}_{2}$ 晶粒具有典型的片状形貌。 $\mathrm{ZrB}_{2}$ 属于六方晶系, 其晶粒在(0001)面的表面能高 于其它面，沿 $<210>$ 及 $<110>$ 方向则具有较低的扩散 活化能，这使得 $\mathrm{ZrB}_{2}$ 沿 $a$ 或 $b$ 轴的生长速度更快， 最终生成片状 $\mathrm{ZrB}_{2}$ 晶粒 ${ }^{[18]}$ 。反应热压烧结过程中, 这些片状 $\mathrm{ZrB}_{2}$ 晶粒在旋转重排过程中互相交错, 形 成了独特的内部互锁微结构, 如图 3(a)中框线内所 示。已有研究证明, 这种特殊的内部互锁结构具有 显著的自强韧效果，有利于复合材料强韧性的改

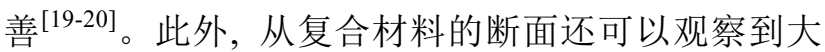
量反应生成的纳米 $\mathrm{SiC}$ 晶粒。这些纳米 $\mathrm{SiC}$ 晶粒主 要分布于 $\mathrm{ZrB}_{2}$ 晶粒周围，或者在 $\mathrm{ZrB}_{2}$ 片状晶粒交 叉所形成的三角区域，或者形成不规则形貌的富 $\mathrm{SiC}$ 区(图 3(a))。在 $\mathrm{ZrB}_{2}-\mathrm{SiC}$ 复合材料中, $\mathrm{SiC}$ 晶粒 尺寸对材料的力学性能具有显著影响, 细小的纳米 $\mathrm{SiC}$ 有利于材料力学性能的提高, 而大尺寸 $\mathrm{SiC}$ 则 更容易成为材料失效的临界缺陷 ${ }^{[21]}$

图 4 为 $\mathrm{C}_{\mathrm{s} f} / \mathrm{ZS}$ 复合材料垂直于热压方向的抛光 面微观形貌。从图 4(a)中可以看出, 采用湿法分散 工艺能有效促进 $\mathrm{C}_{\mathrm{sf}}$ 在陶瓷基体中的均匀分布，没 有明显的纤维团聚现象。此外, 复合材料中 $\mathrm{C}_{\mathrm{sf}}$ 仍保 持较好的原始形貌，这不同于机械球磨法混入短切
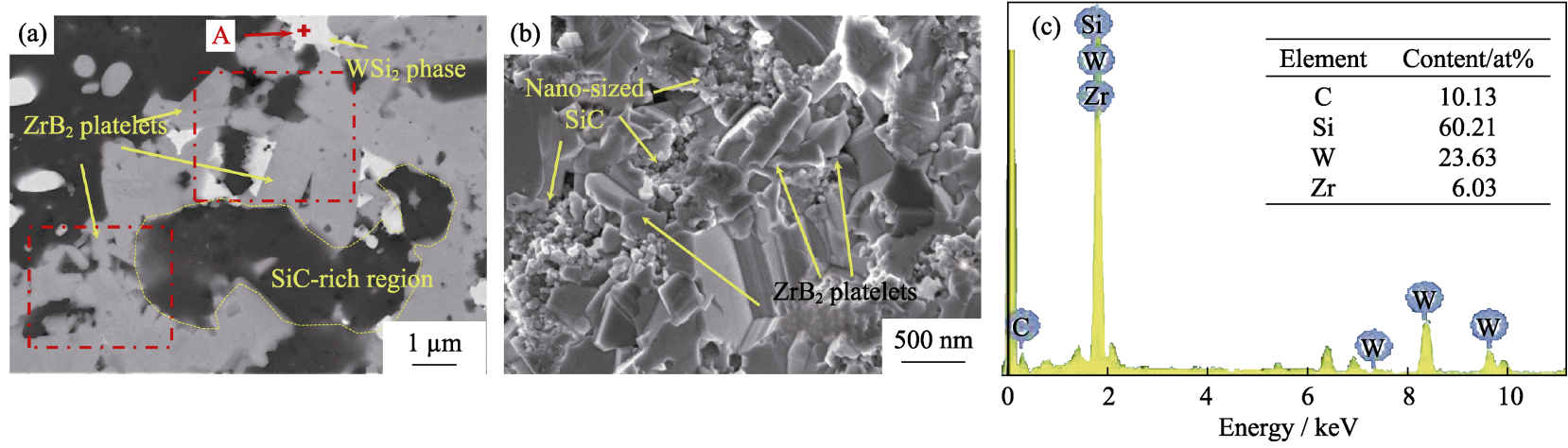

图 3 (a) ZS 样品抛光表面背散射电子显微照片; (b) ZS 样品断面二次电子显微照片; (c) 图 3(a)中 A 点 EDS 分析结果

Fig. 3 (a) Backscattered electron micrograph taken from polished surface of ZS sample, (b) secondary electron micrograph taken from fracture surface of ZS sample, and (c) EDS analysis taken from the point A in (a) 


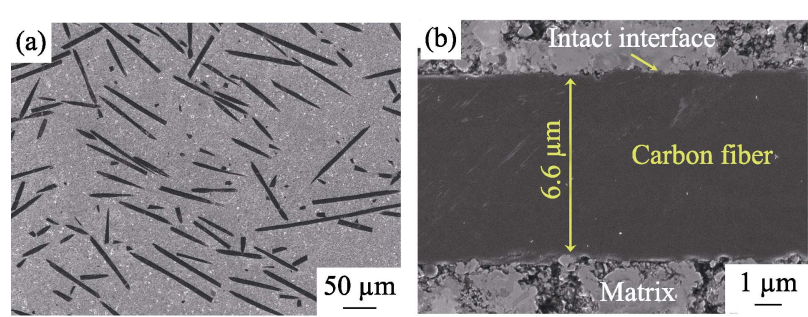

图 $4 \mathrm{C}_{\mathrm{s}} \mathrm{f} / \mathrm{ZS}$ 复合材料电子显微照片

Fig. 4 SEM micrographs of $\mathrm{C}_{\mathrm{sf}} / \mathrm{ZS}$ composites (a) Polished surface; (b) Interfacial domain

纤维, 其往往难以避免机械损伤而产生大量纤维碎 屑, 弱化了纤维的增㓞作用。图 4(b)为 $\mathrm{C}_{\mathrm{sf}} / \mathrm{ZS}$ 样品 中典型的纤维/基体界面微观形貌。 $\mathrm{C}_{\mathrm{sf}}$ 在烧结后仍 具有平整的形貌轮廓, 纤维与基体之间界面清晰, 且纤维沿轴向平滑延伸。 $\mathrm{C}_{\mathrm{sf}}$ 直径均匀, 测量显示约 为 $6.6 \mu \mathrm{m}$, 与碳纤维的原始直径基本一致, 这说明 陶瓷基体在高温环境中对 $\mathrm{C}_{\mathrm{sf}}$ 的侵蚀有限。众所周 知, 碳纤维作为超高温陶瓷基复合材料增韧体所面 临的主要问题是烧结过程中与基体相反应造成纤维 侵蚀降解, 这不仅造成纤维强度降低, 还使纤维与 基体产生较强的化学结合界面, 从而影响碳纤维在 承载过程中发挥相应的能量耗散机制。本研究在较 低烧结温度制备 $\mathrm{C}_{\mathrm{s}} / \mathrm{ZS}$ 复合材料, 减少碳纤维与 $\mathrm{ZrB}_{2}$ 、 $\mathrm{ZrO}_{2}$ 及 $\mathrm{SiO}_{2}$ 等发生反应侵蚀 ${ }^{[9]}$; 同时分散纤维的胶 体粒子在烧结过程中产生裂解炭, 其具有较高活性 参与反应(4), 从而降低了 $\mathrm{C}_{\mathrm{sf}}$ 与 $\mathrm{ZrSi}_{2}$ 反应的可能性, 使得 $\mathrm{C}_{\mathrm{sf}}$ 烧结后呈现出较好的完整性, 避免纤维与 基体形成强结合界面，有利于 $\mathrm{C}_{\mathrm{sf}}$ 发挥主体增韧作用。

\section{4 复合材料力学性能及强韧化机理对比分析}

表 2 列出了 $\mathrm{ZS}$ 及 $\mathrm{C}_{\mathrm{sf}} \mathrm{ZSS}$ 复合材料力学性能测 试结果。可以看到, ZS 复合材料的维氏硬度、弯曲 强度及断裂㓞性分别为 $(17.1 \pm 0.7) \mathrm{GPa} 、(655 \pm 21) \mathrm{MPa}$ 及 $(6.08 \pm 0.17) \mathrm{MPa} \cdot \mathrm{m}^{1 / 2}$ 。复合材料表现出较高的弯 曲强度与断裂韧性, 特别是断裂韧性显著高于传统 $\mathrm{ZrB}_{2}-\mathrm{SiC}$ 陶瓷(3.5 5.0 $\left.\mathrm{MPa} \cdot \mathrm{m}^{1 / 2}\right)$, 这说明材料中形 成的内部互锁微结构具有优异的自强韧效果。类似 地， $\mathrm{Zou}$ 等 ${ }^{[19]}$ 在 $2200{ }^{\circ} \mathrm{C}$ 无压烧结制备的 $\mathrm{ZrB}_{2}-\mathrm{SiC}-\mathrm{WC}$ 陶瓷中, $\mathrm{ZrB}_{2}$ 晶粒取向生长并形成了 互锁微结构, 对比于未形成互锁微结构的 $\mathrm{ZrB}_{2}-\mathrm{SiC}$ 陶瓷, 其断裂韧性由 $5.0 \mathrm{MPa} \cdot \mathrm{m}^{1 / 2}$ 提升至 $6.5 \mathrm{MPa} \cdot \mathrm{m}^{1 / 2}$ 。

表 $2 \mathrm{ZS}$ 及 $\mathrm{C}_{\mathrm{sf}} / \mathrm{ZS}$ 复合材料的力学性能

Table 2 Mechanical properties of $\mathrm{ZS}$ and $\mathrm{C}_{\mathrm{sf}} / \mathrm{ZS}$ composites

\begin{tabular}{cccccc}
\hline Sample & $\begin{array}{c}\text { Vickers } \\
\text { hardness } \\
/ \mathrm{GPa}\end{array}$ & $\begin{array}{c}\text { Flexural } \\
\text { strength, } \\
\sigma / \mathrm{MPa}\end{array}$ & $\begin{array}{c}\text { Fracture } \\
\text { toughness, } \\
K_{\mathrm{IC}} / \\
\left(\mathrm{MPa} \cdot \mathrm{m}^{1 / 2}\right)\end{array}$ & $K_{\mathrm{IC}} / \sigma$ & $\begin{array}{c}\text { Work of } \\
\text { fracture/ } \\
\left(\mathrm{J} \cdot \mathrm{m}^{-2}\right)\end{array}$ \\
\hline $\mathrm{ZS}$ & $(17.1 \pm 0.7)$ & $(655 \pm 21)$ & $(6.08 \pm 0.17)$ & 0.009 & 149.40 \\
$\mathrm{C}_{\mathrm{sf}} / \mathrm{ZS}$ & $(13.8 \pm 0.2)$ & $(368 \pm 27)$ & $(5.41 \pm 0.25)$ & 0.015 & 264.06 \\
\hline
\end{tabular}

对比来看, $\mathrm{C}_{\mathrm{sf}} / \mathrm{ZS}$ 复合材料的维氏硬度、弯曲强 度及断裂韧性分别为(13.8 \pm 0.2$) \mathrm{GPa} 、(368 \pm 27) \mathrm{MPa}$ 及 $(5.41 \pm 0.25) \mathrm{MPa} \cdot \mathrm{m}^{1 / 2}$ 。 $\mathrm{C}_{\mathrm{sf}} / \mathrm{ZrB}_{2}-\mathrm{SiC}$ 复合材料中 $\mathrm{C}_{\mathrm{sf}}$ 增韧作用的发挥受其结构状态影响显著, 严重损 伤的 $\mathrm{C}_{\mathrm{sf}}$ 使 $\mathrm{C}_{\mathrm{sf}} / \mathrm{ZrB}_{2}-\mathrm{SiC}$ 复合材料的断裂韧性局限在 3 4 $\mathrm{MPa} \cdot \mathrm{m}^{1 / 2[10]}$ 。本研究中 $\mathrm{C}_{\mathrm{sf}}$ 保持了较为完整的 结构形貌，从而使 $\mathrm{C}_{\mathrm{s} f} / \mathrm{ZS}$ 材料的断裂韧性达到 $5.41 \mathrm{MPa} \cdot \mathrm{m}^{1 / 2}$ 。但也注意到 $\mathrm{C}_{\mathrm{s}} / \mathrm{ZS}$ 维氏硬度和弯曲 强度低于 $\mathrm{ZS}$, 这与 $\mathrm{C}_{\mathrm{sf}}$ 引入造成材料内部缺陷增加 及缺陷类型改变有关, 同时也与 $\mathrm{C}_{\mathrm{sf}}$ 和基体间弹性 模量失配有关。根据复合材料强度混合准则, 增韧 相弹性模量低于陶瓷基体，将造成复合材料强度的 下降。类似现象也出现在其它碳纤维增韧陶瓷基复 合材料中 ${ }^{[22-23]}$ 。

此外, 对于陶瓷材料而言, 断裂破坏源于裂纹 的非稳态扩展。给定一个材料体系, 则存在一个临 界裂纹尺寸 $\alpha_{\mathrm{cr}}$, 当裂纹尺寸大于 $\alpha_{\mathrm{cr}}$ 时, 材料在承载 或热冲击过程中该裂纹就容易发生失稳扩张，从而 造成材料断裂性能大幅降低 ${ }^{[24]}$ 。因此材料的临界裂 纹尺寸越大, 材料的裂纹尺寸容限值就越大, 有利 于材料损伤容限的提升。式(5)为临界裂纹尺寸 $\alpha_{\mathrm{cr}}$ 的表达式 ${ }^{[9]}$ :

$$
\alpha_{\mathrm{cr}}=K_{\mathrm{IC}}^{2} / \pi \sigma^{2}
$$

其中, $K_{\mathrm{IC}}$ 为材料的断裂韧性, $\sigma$ 为材料的弯曲强度。 材料的临界裂纹尺寸与 $K_{\mathrm{IC}}$ 和 $\sigma$ 的比值相关, $K_{\mathrm{IC}} / \sigma$ 的微小提升将大幅提高复合材料的临界裂纹尺寸。 如表 2 中所示, $\mathrm{C}_{\mathrm{s} f} / \mathrm{ZS}$ 复合材料的 $K_{\mathrm{IC}} / \sigma$ 比 $\mathrm{ZS}$ 明显 提升, 说明 $\mathrm{C}_{\mathrm{sf}}$ 的引入可以显著增大材料的临界裂 纹尺寸，使复合材料具有更大的损伤容限。

图 5 为 $\mathrm{ZS}$ 与 $\mathrm{C}_{\mathrm{s} f} / \mathrm{ZS}$ 样品在 SENB 测试中获取 的载荷-位移曲线。对于 $\mathrm{ZS}$ 复合材料, 载荷随位移 线性增加至最高值后瞬间下降，材料失效是典型的 脆性破坏模式。与之不同, $\mathrm{C}_{\mathrm{sf}} / \mathrm{ZS}$ 复合材料在载荷到 达最高值附近出现密集的载荷波动, 之后载荷出现 多个阶梯状下降直至材料最终失效。这说明 $\mathrm{C}_{\mathrm{sf}} / \mathrm{ZS}$ 材料在达到承载极限后, 裂纹并没有迅速失稳, 而 是呈现出半稳定扩展，这也与 $\mathrm{C}_{\mathrm{s}} \mathrm{f} / \mathrm{ZS}$ 复合材料具有 更高的裂纹尺寸容限有关。同时, 如图 5 中宏观测 试样条所示, 与 $\mathrm{ZS}$ 样品不同, $\mathrm{C}_{\mathrm{s} f} / \mathrm{ZS}$ 样品在完成 SENB 测试后并未完全断开, 表现出一种典型的非 灾难性破坏。

另外, 从 SENB 测试曲线中可以观察到, $\mathrm{C}_{\mathrm{sf}} / \mathrm{ZS}$ 样品的失效位移比 ZS 样品显著增加。复合材料的 断裂㓞性由 SENB 测试中的力峰值计算获得, 其表 征了材料对裂纹开始扩展的抵抗能力; 而断裂功 


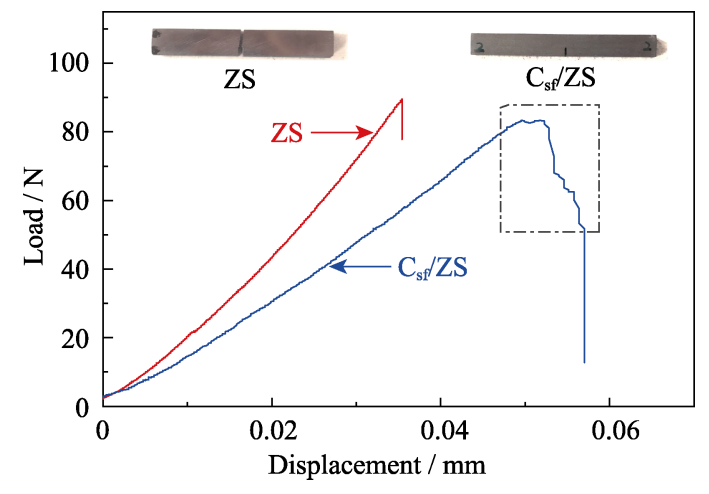

图 $5 \mathrm{ZS}$ 与 $\mathrm{C}_{\mathrm{sf}} / \mathrm{ZS}$ 复合材料的 $\mathrm{SENB}$ 测试载荷-位移曲线 Fig. 5 Load-displacement curves of $\mathrm{ZS}$ and $\mathrm{C}_{\mathrm{sf}} / \mathrm{ZS}$ composites during SENB tests

$\gamma_{\text {wof }}$ 由 SENB 测试中载荷-位移曲线下方包络面积计 算获得, 表征了材料对整个裂纹扩展过程的抵抗能 力。当 SENB 测试中力峰值差距不大时, $\mathrm{C}_{\mathrm{s}} / \mathrm{ZS}$ 样品 更大的失效位移说明材料在失效之前消耗了更多的 外力做功。计算结果显示, $\mathrm{ZS}$ 与 $\mathrm{C}_{\mathrm{sf}} / \mathrm{ZS}$ 的断裂功分 别为 149.40 和 $264.06 \mathrm{~J} / \mathrm{m}^{2}, \mathrm{C}_{\mathrm{sf}} / \mathrm{ZS}$ 复合材料的断裂 功提高了 $76.7 \%$, 说明承载过程中, $\mathrm{C}_{\mathrm{sf}} / \mathrm{ZS}$ 在完全破 坏之前消耗掉了更多的能量, 引入 $\mathrm{C}_{\mathrm{sf}}$ 有效提高了 复合材料抵抗裂纹扩展的能力。

为了进一步探究造成 $\mathrm{ZS}$ 及 $\mathrm{C}_{\mathrm{s} f} / \mathrm{ZS}$ 复合材料断 裂行为差异的机理, 对两种材料进行了断面及裂纹 扩展分析。图 6 为 ZS 复合材料断面及抛光表面的 裂纹扩展 SEM 照片。ZS 样品断面处可以观察到片 状的 $\mathrm{ZrB}_{2}$ 晶粒和大量的纳米 $\mathrm{SiC}$, 以及由 $\mathrm{ZrB}_{2}$ 晶粒 形成的互锁结构(图 6(a))。互锁结构中较大的 $\mathrm{ZrB}_{2}$ 晶粒以穿晶断裂为主, 而小尺寸的 $\mathrm{ZrB}_{2}$ 晶粒则表现 为沿晶断裂模式。纳米 $\mathrm{SiC}$ 则多填充于 $\mathrm{ZrB}_{2}$ 晶粒周 围, 主要为沿晶断裂模式。从 $\mathrm{ZS}$ 材料裂纹扩展路径 (图 6(b)) 可以看出, 材料展现出大量的裂纹偏折现 象, 特别是在扩展裂纹遇到片状 $\mathrm{ZrB}_{2}$ 晶粒形成的互 锁微结构时, 裂纹发生偏折, 同时片状 $\mathrm{ZrB}_{2}$ 沿晶断 裂形成了一定的裂纹桥接, 使裂纹扩展路径增加, 提高了材料的断裂韧性。这种内部互锁微结构以及 混合断裂模式是 ZS 复合材料表现出优异力学性能 的主要原因。

图 7 为 $\mathrm{C}_{\mathrm{s} f} / \mathrm{ZS}$ 复合材料断面及抛光表面裂纹扩 展 SEM 照片。从 $\mathrm{C}_{\mathrm{s} f} / \mathrm{ZS}$ 样品断面可以看到碳纤维 横截面呈现出完好的圆形轮廓, 纤维表面完整, 无 明显腐蚀损伤迹象。此外, 材料断面可以观察到大 量的纤维拔出及拔出后形成的孔洞(图 7(a))。表明碳 纤维可以有效发挥拔出、脱粘和摩擦等能量耗散机 理，提高复合材料对裂纹扩展的抵抗能力。对 $\mathrm{C}_{\mathrm{sf}} / \mathrm{ZS}$ 样品的基体进行放大观察, 可以看出基体中存在大 量的纳米颗粒(图 7(b)), 这些纳米颗粒主要是 $\mathrm{SiC}$ 及

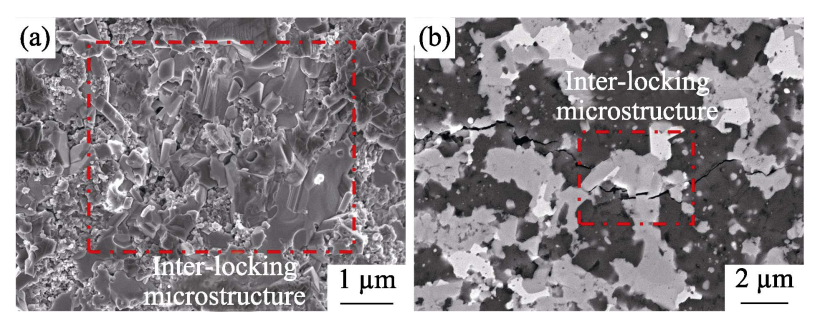

图 6 (a) ZS 样品断面二次电子显微照片; (b) ZS 样品表面裂 纹扩展背散射电子显微照片

Fig. 6 (a) Secondary electron micrograph taken from fracture surface of ZS sample, and (b) backscattered electron micrograph of indentation crack propagation on polished surface of ZS sample
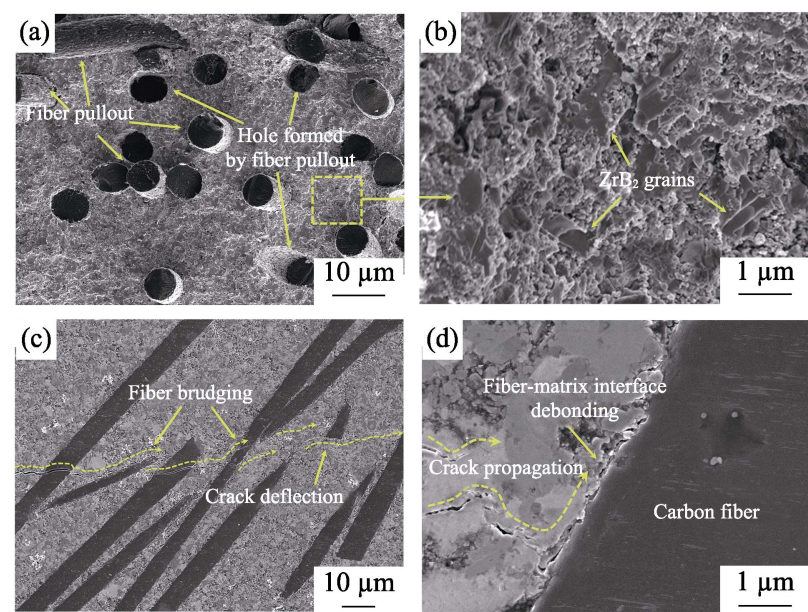

图 $7 \mathrm{C}_{\mathrm{s} f} / \mathrm{ZS}$ 复合材料的 SEM 照片

Fig. 7 SEM micrographs of $\mathrm{C}_{\mathrm{sf}} / \mathrm{ZS}$ composites (a) Fracture surface; (b) Matrix; (c, d) Crack propagation path

$\mathrm{ZrC}$ 。同时，依旧可以看到较大尺寸的片状 $\mathrm{ZrB}_{2}$ 晶 粒，但不同于 $\mathrm{ZS}$ 复合材料, $\mathrm{C}_{\mathrm{sf}} / \mathrm{ZS}$ 中很难观察到片 状 $\mathrm{ZrB}_{2}$ 晶粒形成的内部互锁结构。这可能是由于 $\mathrm{ZrSi}_{2}$ 与裂解碳反应造成了烧结过程中液相减少，从 而影响 $\mathrm{ZrB}_{2}$ 晶粒在压力作用下的排列与生长，同时 生成的大量纳米颗粒又进一步制约了 $\mathrm{ZrB}_{2}$ 晶粒的 取向生长。因此, $\mathrm{C}_{\mathrm{sf}} \mathrm{Z} / \mathrm{ZS}$ 样品中基体内互锁结构的减 少也是材料弯曲强度降低的一个原因。

另外，不同于图 6(b)中 ZS 样品表面的裂纹扩展 路径，图 7(c)中 $\mathrm{C}_{\mathrm{sf}} / \mathrm{ZS}$ 样品表面的裂纹扩展路径呈 现出一种明显的不连续现象, 这也与两种材料不同 的断裂模式相对应。由于具有高长径比 $\mathrm{C}_{\mathrm{sf}}$ 的存在, 裂纹在扩展过程中必须切断纤维或者沿纤维/基体 界面传递。本研究中, $\mathrm{C}_{\mathrm{sf}}$ 保持了较好的完整性, 裂 纹在扩展至纤维附近时, 受到有效阻拦而产生大量 分叉，这些分叉裂纹沿纤维/基体界面不断扩展，直 至发生纤维脱粘，从而造成裂纹扩展路径的不连续, 如图 7(d)所示。脱粘后的纤维通过拔出及界面摩擦 进一步消耗裂纹扩展能量, 造成复合材料裂纹半稳 态扩展, 从而断裂功明显增加, 使得破坏模式转变 为非灾难性断裂破坏。 
通过上面的对比分析可以看到，反应热压烧结 制备的 ZS 复合材料中形成了大量的片状 $\mathrm{ZrB}_{2}$ 晶粒 以及互锁微结构, 呈现出较好的自强韧化效果, 材 料的弯曲强度及断裂韧性较高, 但临界裂纹尺寸及 断裂功较低, 并且表现出典型的陶瓷材料脆性断裂 特征; 以 $\mathrm{C}_{\mathrm{sf}}$ 作为增韧相, $\mathrm{C}_{\mathrm{sf}} / \mathrm{ZS}$ 复合材料中片状 $\mathrm{ZrB}_{2}$ 晶粒形成的互锁微结构减少, 材料弯曲强度下 降, 但高长径比的 $\mathrm{C}_{\mathrm{sf}}$ 表现出显著的增韧作用, 材料 不仅具有较高的断裂韧性, 同时临界裂纹尺寸及断 裂功都显著提高, 表现出了非灾难性破坏模式。

\section{3 结论}

以 $\mathrm{ZrSi}_{2} 、 \mathrm{~B}_{4} \mathrm{C}$ 及 $\mathrm{C}$ 为原料, 通过反应热压烧结 制备了 $\mathrm{ZrB}_{2}-\mathrm{SiC}$ 复合材料, 并进一步引入 $\mathrm{C}_{\mathrm{sf}}$ 作为 增韧相制备了 $\mathrm{C}_{\mathrm{sf}} / \mathrm{ZrB}_{2}-\mathrm{SiC}$ 陶瓷基复合材料, 对比 分析了两种复合材料微观结构、力学性能及强韧化 机理, 得到如下结论:

1) 反应热压烧结制备的 $\mathrm{ZrB}_{2}-\mathrm{SiC}$ 复合材料中 生成了大量的纳米 $\mathrm{SiC}$, 而 $\mathrm{ZrB}_{2}$ 生长为片状晶粒, 且互相交错, 在复合材料中形成了特殊的互锁微 结构。

2) 在 $\mathrm{C}_{\mathrm{sf}} / \mathrm{ZrB}_{2}-\mathrm{SiC}$ 复合材料基体中纳米 $\mathrm{SiC}$ 及 $\mathrm{ZrC}$ 含量增加, 且片状 $\mathrm{ZrB}_{2}$ 晶粒形成的互锁微结构 减少, $\mathrm{C}_{\mathrm{sf}}$ 没有受到明显的基体侵蚀, 保持了较为完 整的微观形貌。

3) 片状 $\mathrm{ZrB}_{2}$ 晶粒形成的互锁微结构展现了优 异的自强韧化效果, $\mathrm{ZrB}_{2}-\mathrm{SiC}$ 复合材料具有较高的 弯曲强度及断裂韧性, 但材料表现出典型的脆性断 裂特征; $\mathrm{C}_{\mathrm{sf}} / \mathrm{ZrB}_{2}-\mathrm{SiC}$ 复合材料中 $\mathrm{C}_{\mathrm{sf}}$ 表现出显著的 增韧作用，材料不仅具有较高的断裂韧性，同时临 界裂纹尺寸及断裂功都大幅提高, 表现出非灾难性 破坏模式。

\section{参考文献:}

[1] FAHRENHOLTZ W G, HiLMAS G E, TALMY I G, et al. Refractory diborides of zirconium and hafnium. Journal of the American Ceramic Society, 2007, 90(5): 1347-1364.

[2] CHAMBERLAIN A L, FAHRENHOLTZ W G, HILMAS G E, et al. High-strength zirconium diboride-based ceramics. Journal of the American Ceramic Society, 2004, 87(6): 1170-1172.

[3] GUO S Q. Densification of $\mathrm{ZrB}_{2}$-based composites and their mechanical and physical properties: a review. Journal of the European Ceramic Society, 2009, 29(6): 995-1011.

[4] ZHENG H Y, MENG C X, HU D L, et al. EBSD Analysis for orientation relationship of textured $\mathrm{ZrB}_{2}-\mathrm{SiC}$ ultra-high temperature ceramics. Journal of Inorganic Materials, 2018, 33(4): 380-384.

[5] ZHANG G J, NI D W, ZOU J, et al. Inherent anisotropy in transition metal diborides and microstructure/property tailoring in ultrahigh temperature ceramics-a review. Journal of the European Ceramic Society, 2017, 38(2): 371-389.
[6] WU W W, WANG Z, ZHANG G J, et al. $\mathrm{ZrB}_{2}-\mathrm{MoSi}_{2}$ composites toughened by elongated $\mathrm{ZrB}_{2}$ grains via reactive hot pressing. Scripta Materialia, 2009, 61(3): 316-319.

[7] SHAHEDI ASL M, FARAHBAKHSH I, NAYEBI B. Characteristics of multi-walled carbon nanotube toughened $\mathrm{ZrB}_{2}-\mathrm{SiC}$ ceramic composite prepared by hot pressing. Ceramics International, 2016, 42(1): 1950-1958.

[8] ZHU T, XU L, ZHANG X H, et al. Densification, microstructure and mechanical properties of $\mathrm{ZrB}_{2}-\mathrm{SiC}_{\mathrm{w}}$ ceramic composites. Journal of the European Ceramic Society, 2009, 29(13): 2893-2901.

[9] HU P, GUI K X, HONG W H, et al. High-performance $\mathrm{ZrB}_{2}-\mathrm{SiC}-\mathrm{C}_{\mathrm{f}}$ composite prepared by low-temperature hot pressing using nanosized $\mathrm{ZrB}_{2}$ powder. Journal of the European Ceramic Society, 2017, 37(6): 2317-2324.

[10] GUI K X, LIU F Y, WANG G, et al. Inhibited degradation of carbon fibers in $\mathrm{ZrB}_{2}-\mathrm{SiC}-\mathrm{C}_{\mathrm{sf}}$ ultra-high temperature ceramic composites. Journal of Synthetic Crystals, 2018, 47(2): 418-423.

[11] HE P G, JIA D C, LI Y T, et al. Preparation and mechanical performance of ductile $\mathrm{C}_{\mathrm{s} f} / \mathrm{Al}_{2} \mathrm{O}_{3}$-BN composites-Part 2: Effects of fiber contents and ablation properties. Ceramics International, 2016, 42(9): 11063-11069.

[12] BAI Y L, HE X D, ZHU C C, et al. Microstructures, electrical, thermal, and mechanical properties of bulk $\mathrm{Ti}_{2} \mathrm{AlC}$ synthesized by self-propagating high-temperature combustion synthesis with pseudo hot isostatic pressing. Journal of the American Ceramic Society, 2012, 95(1): 358-364.

[13] WANG M C, ZHANG Z G, SUN Z J, et al. Effect of fiber type on mechanical properties of short carbon fiber reinforced $\mathrm{B}_{4} \mathrm{C}$ composites. Ceramics International, 2009, 35(4): 1461-1466.

[14] ZU Y F, SHA J J, LI J, et al. Effect of multi-walled carbon nanotubes on microstructure and fracture properties of carbon fiberreinforced $\mathrm{ZrB}_{2}$-based ceramic composite. Ceramics International, 2017, 43(10): 7454-7460.

[15] SHA J J, ZHANG Z F, DI S X, et al. Microstructure and mechanical properties of $\mathrm{ZrB}_{2}$-based ceramic composites with nano-sized $\mathrm{SiC}$ particles synthesized by in-situ reaction. Materials Science and Engineering A, 2017, 693: 145-150.

[16] SILVESTRONI L, MERIGGI G, SCITI D. Oxidation behavior of $\mathrm{ZrB}_{2}$ composites doped with various transition metal silicides. Corrosion Science, 2014, 83: 281-291.

[17] LIU H L, LIU J X, LIU H T, et al. Changed oxidation behavior of $\mathrm{ZrB}_{2}$-SiC ceramics with the addition of $\mathrm{ZrC}$. Ceramics International, 2015, 41(6): 8247-8251.

[18] HU C F, ZOU J, HUANG Q, et al. Synthesis of plate-Like $\mathrm{ZrB}_{2}$ Grains. Journal of the American Ceramic Society, 2012, 95(1): 85-88.

[19] ZOU J, ZHANG G J, KAN Y M. Formation of tough interlocking microstructure in $\mathrm{ZrB}_{2}$-SiC-based ultrahigh-temperature ceramics by pressureless sintering. Journal of Materials Research, 2009, 24(7): 2428-2434.

[20] XU L, HUANG C Z, LIU H L, et al. In situ synthesis of $\mathrm{ZrB}_{2}$ $-\mathrm{ZrC}_{x}$ ceramic tool materials toughened by elongated $\mathrm{ZrB}_{2}$ grains. Materials \& Design, 2013, 49: 226-233.

[21] ZHANG S C, HILMAS G E, FAHRENHOLTZ W G. Mechanical properties of sintered $\mathrm{ZrB}_{2}$-SiC ceramics. Journal of the European Ceramic Society, 2011, 31(5): 893-901.

[22] YANG F Y, ZHANG X H, HAN J C, et al. Analysis of the mechanical properties in short carbon fiber-toughened $\mathrm{ZrB}_{2}-\mathrm{SiC}$ ultrahigh temperature ceramic. Journal of Composite Materials, 2010, 44(8): 953-961.

[23] VINCI A, ZOLI L, SCITI D, et al. Understanding the mechanical properties of novel UHTCMCs through random forest and regression tree analysis. Materials \& Design, 2018, 145: 97-107.

[24] TAYLOR D, CORNETTI P, PUGNO N. The fracture mechanics of finite crack extension. Engineering Fracture Mechanics, 2005, 72(7): 1021-1038. 